\title{
Enterocolitis necrosante: análisis retrospectivo de 1428 recién nacidos prematuros en una unidad de cuidados intensivos neonatales de nivel III durante un período de cuatro años
}

Developing necrotizing enterocolitis: retrospective analysis of 1428 preterm infants at a level-III neonatal intensive care unit over a four years period

\section{Prof. Asoc. Dr. Hakan Ongun ${ }^{a}$ Dr. Selim Demirezen ${ }^{b}$ y Prof. Asoc. Dra. Meltem Demirc}

\section{RESUMEN}

Objetivo. Investigar la frecuencia de la enterocolitis necrosante (ECN) en prematuros y evaluar los factores de riesgo asociados con su aparición y progresión a perforación intestinal. Métodos. Cohorte retrospectiva de prematuros hospitalizados entre 2015 y 2018. Las características perinatales, clínicas, nutricionales y de laboratorio se analizaron con SPSS-23, y los factores de riesgo mediante una regresión logística.

Resultados. En 1428 neonatos, la tasa de ECN fue del $18,28 \%$. La concepción por reproducción asistida, la cesárea y los corticoesteroides posnatales se asociaron con ECN (OR: 4,056; IC del $95 \%$ : 2,810-5,854; OR: 1,961; IC del $95 \%$ : 1,321-2,910; OR: 6,422; IC del $95 \%$ : 4,327-9,530). El momento de la primera alimentación enteral se asoció con ECN, pero no con perforación intestinal $(p<0,001 ; p=0,604)$. De 261 pacientes con ECN, $47(18 \%)$ tuvieron perforación intestinal.Elmomento dela primera alimentación enteral y el ductus persistente con repercusión hemodinámica fueron factores predisponentes para ECN, y el puntaje de Apgar bajo al minuto 5 para perforación intestinal. (OR: 6,515; IC del $95 \%$ : 5,011-8,470; OR: 4,715; IC del $95 \%$ : 2,7178,183; OR: 2,748; IC del $95 \%$ : 1,100-6,866). La mortalidad fue del $9 \%$. La ECN aumentó el riesgo de mortalidad 2,192 veces (IC del $95 \%$ : 1,469-3,271); en perforación intestinal, el riesgo aumentó 11,527 veces (IC del 95\%:6,293-21,115). Conclusión. El conducto arterial persistente y el retraso en la primera nutrición enteral fueron factores predisponentes para ECN, y el puntaje de Apgar bajo al minuto 5 para perforación intestinal.

Palabras clave: enterocolitis necrosante, nutrición, reciénnacido prematuro, perforación intestinal, puntaje de Apgar.

http:/ / dx.doi.org/10.5546/ aap.2020.405

Texto completo en inglés:

http: / / dx.doi.org/10.5546/ aap.2020.eng.405

Cómo citar: Ongun H, Demirezen S, Demir M Enterocolitis necrosante: análisis retrospectivo de 1428 recién nacidos prematuros en una unidad de cuidados intensivos neonatales de nivel III durante un período de cuatro años. Arch Argent Pediatr 2020;118(6):405-410.

\section{INTRODUCCIÓN}

La nutrición enteral adecuada es indispensable en el tratamiento de los recién nacidos prematuros para evitar las complicaciones asociadas con el acceso vascular, la sepsis y los efectos adversos de la nutrición parenteral. ${ }^{1,2}$ La demora en lograr la nutrición total puede causar disfunción intestinal. ${ }^{3}$ Sin embargo, establecer la nutrición enteral total en un recién nacido prematuro es más difícil de lo previsto y puede llevar de una a dos semanas, según el estado de salud del recién nacido. ${ }^{4} \mathrm{La}$ inmadurez intestinal y las comorbilidades relacionadas con la prematuridad contribuyen a la aparición de problemas de alimentación y complicaciones intestinales, incluida la enterocolitis necrosante (ECN).

La ECN es una de las enfermedades más destructivas que padecen los recién nacidos prematuros. ${ }^{3-6}$ La prevalencia es de 1 a 3 por cada 1000 nacidos vivos y representa el 7,7\% del total de ingresos en las unidades de cuidados intensivos neonatales (UCIN). ${ }^{5} \mathrm{La}$ incidencia aumenta cuanto más temprana es la edad gestacional (EG) y menor es el peso al nacer. ${ }^{6,7}$

La patogenia sigue siendo multifactorial, con diferentes procesos patológicos y varios endotipos. ${ }^{3,6} \mathrm{Se}$ considera que la inmadurez intestinal, la barrera inadecuada de la mucosa, la disbiosis microbiana y la inestabilidad circulatoria del tubo digestivo cumplen una función en el mecanismo patológico. ${ }^{3}$ Las manifestaciones clínicas incluyen distensión abdominal, vómitos biliosos, residuos gástricos, heces con sangre y parámetros fisiológicos anómalos, como apnea, 
bradicardia, alteraciones hemodinámicas e inestabilidad térmica. ${ }^{3,8}$

Se calcula que la mortalidad es del $10 \%$ al $40 \%$, pero la perforación intestinal y las complicaciones secundarias (infecciones graves, estenosis, complicaciones del estoma y síndrome de intestino corto) aumentan significativamente la mortalidad al $76 \%{ }^{3,9,10}$ A pesar de la mayor supervivencia de los neonatos con muy bajo peso al nacer, se ha progresado muy poco en el tratamiento de los pacientes con ECN. ${ }^{1}$ Dichas consecuencias exigen actuar con suma cautela frente a estos recién nacidos, que corren riesgo de presentar problemas intestinales. Es crucial identificar la ECN de forma temprana, porque, una vez que comienza, el proceso patológico es agresivo y difícil de detener. El objetivo fue investigar la frecuencia de la ECN en una población de prematuros de una UCIN de alta complejidad, que recibe muchas derivaciones y brinda servicios en un territorio densamente poblado en torno a la región mediterránea, además de evaluar los factores de riesgo asociados con el inicio de la enfermedad y la progresión a la perforación intestinal.

\section{POBLACIÓN Y MÉTODOS}

La cohorte retrospectiva y unicéntrica incluyó a recién nacidos prematuros (edad gestacional menor de 37 semanas) que ingresaron entre 2015 y 2018. El comité de ética de la institución aprobó el estudio.

La UCIN es un centro de alta complejidad, con 34 camas, afiliado a la universidad. Brinda atención neonatal bajo la supervisión de neonatólogos con certificado de especialidad, y el cociente de recursos de enfermería es de 1:4-1:5 enfermeros / pacientes. Las tasas de ingreso anual son de 850 a 1100 recién nacidos por año, y los prematuros constituyen del $35 \%$ al $50 \%$ de los ingresos. La población de pacientes consiste en neonatos con edad gestacional $\geq 23$ semanas, que tienen anomalías congénitas graves o que requieren una intervención quirúrgica (cardíaca, neuroquirúrgica, abdominal). El enfoque multidisciplinario (subespecialidades pediátricas y especialistas en cirugía) ha convertido a esta UCIN en uno de los centros que reciben más derivaciones. Aproximadamente la mitad de los ingresos son traslados posnatales.

Los datos de los pacientes se obtuvieron en la base de datos del hospital y se preseleccionaron según los códigos de la CIE "prematuridad" y "ECN". Los criterios de exclusión fueron anomalías intestinales (p. ej., peritonitis meconial, vólvulo, atresia) y perforaciones intestinales espontáneas confirmadas por laparotomía. ${ }^{11}$

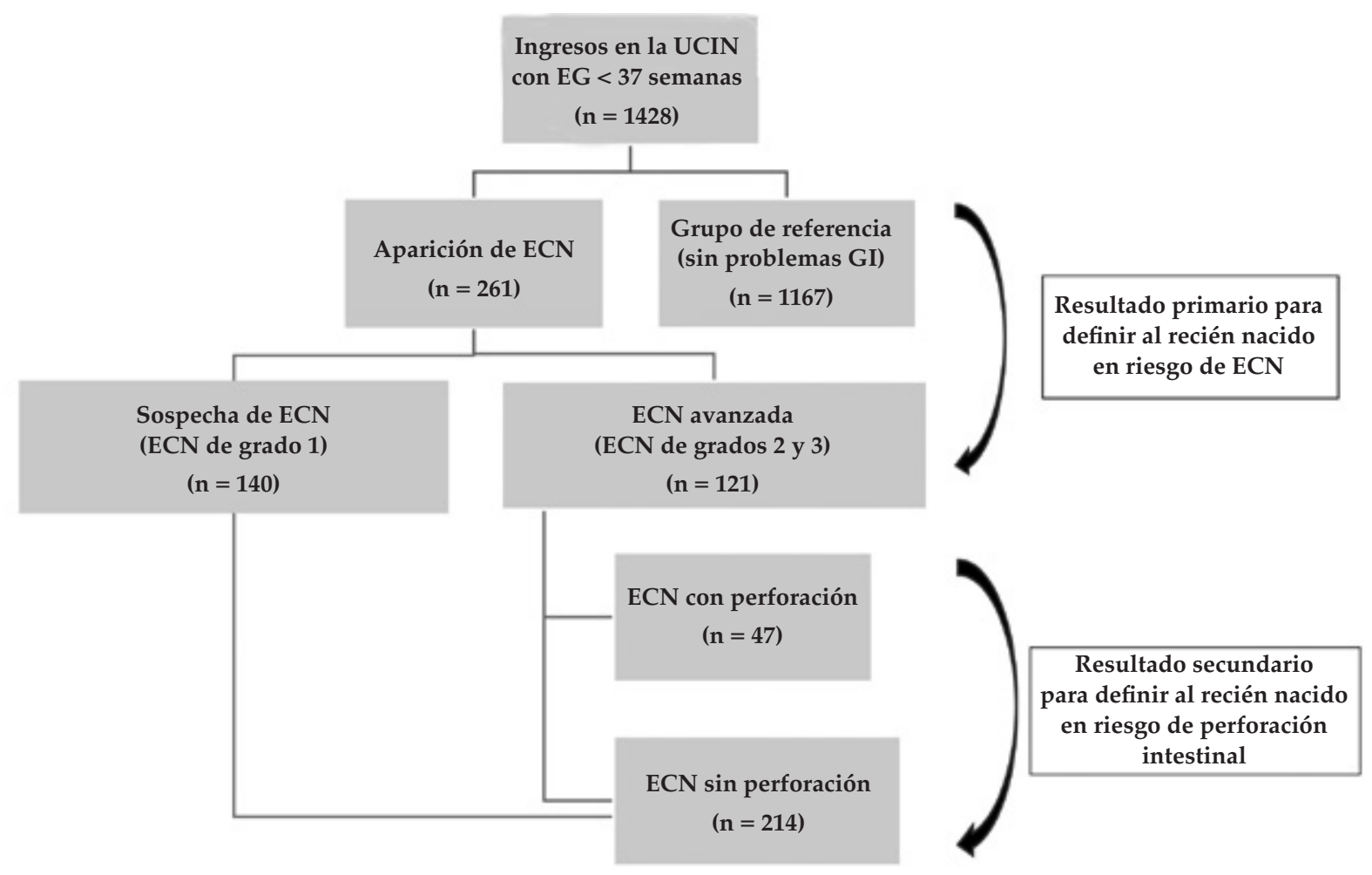


Se dividió a los pacientes en dos grupos principales: recién nacidos con ECN (definida según los criterios de Bell modificados) ${ }^{8}$ y grupo de referencia (recién nacidos sin problemas intestinales). Las subcategorizaciones de los pacientes con ECN se fundaron en (i) la gravedad de la enfermedad (sospecha de ECN: ECN de grado 1, ECN avanzada: ECN de grado 2 y 3) y (ii) la presencia de perforación intestinal confirmada por radiografía y cirugía (perforación intestinal frente a ausencia de perforación).

Se investigaron las características perinatales, las manifestaciones clínicas y los resultados de laboratorio. Se extrajeron de las historias clínicas los datos sobre síndrome de dificultad respiratoria (SDR), ventilación mecánica invasiva (VMI) y asistencia respiratoria no invasiva (presión positiva continua [PPC] nasal), conducto arterial persistente con repercusión hemodinámica (CAP confirmado mediante ecocardiografía, que requirió cirugía o la administración de ibuprofeno), cardiopatía congénita (CC) que no sea $\mathrm{CAP}$, hemorragia intraventricular (HIV) y sepsis (definida según los criterios de Vermont Oxford). ${ }^{12}$ Se evaluaron los hemogramas completos y los análisis de proteína C-reactiva (PCR) y de gases en sangre obtenidos el día de los síntomas iniciales para detectar los primeros signos de anomalías.

Se brindó nutrición enteral a todos los neonatos conforme al protocolo de nutrición institucional (véase el Apéndice del protocolo basado en la pauta de la Sociedad Turca de Neonatología). ${ }^{13}$ Se registraron las variables nutricionales, entre ellas el momento de la primera alimentación enteral, el uso de leche maternizada para prematuros, la edad de inicio de los primeros síntomas intestinales, la duración del ayuno y la nutrición parenteral. Es necesario mencionar que las leyes turcas no regulan el uso de leche donada cuando no se dispone de leche de la madre, situación en la que se utiliza leche maternizada sin diluir para prematuros.

Se utilizó el programa SPSS-23 para el análisis descriptivo de las variables, la tendencia central y la dispersión de las variables cuantitativas. Tras determinar la normalidad (prueba de KolmogorovSmirnov), se utilizó la prueba de la U de MannWhitney o la prueba de la $t$ de Student para analizar la diferencia entre los dos grupos; se realizó el análisis de varianza unifactorial o de Kruskal-Wallis (seguido de comparaciones post hoc mediante la prueba de Bonferroni o Tamhane) para el análisis entre tres grupos. Se efectuaron regresiones logísticas para predecir la probabilidad de padecer ECN y perforación intestinal. Las variables se expresaron como porcentaje $(\%)$, media \pm desviación estándar (DE) o mediana (intervalo intercuartílico), razón de posibilidades (OR) e intervalos de confianza (IC) del $95 \%$. Un valor de $p<0,05$ se consideró significativo.

\section{RESULTADOS}

De 1428 recién nacidos prematuros, $261(18,28 \%)$ tuvieron diagnóstico de ECN. En la Figura 1 se presenta el diagrama de flujo de la población del estudio. Los traslados posnatales representaron el 32,1\% de los pacientes con sospecha de ECN y el $43 \%$ de los pacientes con ECN avanzada. Muchas de las características perinatales no revelaron ninguna significancia (Anexos 1 y 2 en formato electrónico). El tamaño pequeño para la edad gestacional (PEG) y la edad joven de la madre se asociaron con sospecha de ECN $(p=0,033 ; p=0,005)$. Por otro lado, la cesárea fue el tipo de parto más frecuente en los recién nacidos con ECN avanzada $(p=0,001)$. La concepción mediante técnicas de reproducción asistida, la cesárea y la administración de corticoesteroides posnatales se asociaron con la aparición de ECN (OR: 4,056; IC del 95 \%: 2,8105,854; OR: 1,961; IC del $95 \%$ : 1,321-2,910; y OR: 6,422; IC del $95 \%$ : 4,327-9,530, respectivamente). De forma inversa, los corticoesteroides prenatales se asociaron con una tasa más baja de ECN avanzada $(p=0,001)$, pero la influencia no fue clara para la etapa leve de la enfermedad $(p=0,157$ para la sospecha de ECN). El momento de la primera alimentación enteral y el CAP con repercusión hemodinámica fueron los factores predisponentes más determinantes de la ECN (OR: 6,515; IC del $95 \%$ : 5,011-8,470; y OR: 4,715; IC del $95 \%$ : 2,717-8,183; Tabla 1). Los recién nacidos con ECN avanzada tuvieron resultados anómalos en los análisis clínicos, por ejemplo, trombocitopenia y leucocitosis significativas, en las etapas iniciales de los síntomas intestinales ( $p=0,003 ; p<0,001)$.

Cuarenta y siete $(18 \%)$ neonatos presentaron perforación intestinal. En el Anexo 3 (véase en formato electrónico) se muestran las características perinatales basadas en las perforaciones intestinales. En el análisis univariado, el peso al nacer de menos de 750 gramos, la ventilación mecánica invasiva y el puntaje de Apgar bajo al minuto 5 se relacionaron con la mayor probabilidad de padecer una perforación intestinal (OR: 12,010; IC del $95 \%$ : 5,760-25,080; OR: 10,851; IC del $95 \%$ : 3,267-36,045; OR: 4,827; IC del $95 \%$ : 2,439-9,552). Aunque se haya demostrado el vínculo entre la primera alimentación enteral y la aparición de ECN, el impacto de la primera nutrición enteral no fue claro en el caso de la perforación intestinal $(p=0,604)$. Por el contrario, la edad de aparición de los síntomas intestinales iniciales ha indicado una colinealidad con la gravedad de la enfermedad (sospecha de ECN: 13 días; ECN avanzada: 18 días; perforaciones: 19 días). Después del ajuste en función del peso al nacer, el puntaje de Apgar bajo 
al minuto 5 se identificó como el único factor de riesgo independiente de la perforación intestinal (OR: 2,748; IC del $95 \%$ : 1,100-6,866; Tabla 2).

La tasa global de mortalidad fue del $9 \%$. La mortalidad aumentó en los recién nacidos que empeoraron y presentaron ECN avanzada y perforación intestinal $(27,3 \%, 48,9 \%)$. En los recién nacidos prematuros, la ECN aumentó el riesgo de mortalidad 2,192 veces (IC del $95 \%$ : 1,469-3,271). En el caso de la perforación intestinal, la probabilidad aumentó a 11,527 (IC del $95 \%$ : 6,293-21,115).

\section{DISCUSIÓN}

Los avances en la atención neonatal aumentaron la supervivencia en el nacimiento de prematuros; sin embargo, la situación no es la misma cuando se trata de enfermedades gastrointestinales. ${ }^{14,15}$ La evaluación de 1428 recién nacidos prematuros ha demostrado lo siguiente: i) $18,3 \%$ de frecuencia de ECN y 8,5\% de progresión a la enfermedad grave, ii) el tipo de concepción y la cesárea se asociaron con la aparición de ECN, iii) el momento de la primera alimentación enteral y el CAP con repercusión hemodinámica se identificaron como factores de riesgo de la enfermedad, iv) las perforaciones intestinales se produjeron principalmente con un peso al nacer de $<750$ gramos, v) el puntaje de Apgar bajo al minuto 5 fue el único factor de predicción independiente de la perforación intestinal.

Para lograr un progreso en el tratamiento de la ECN, primero debemos comprender la fisiopatología de la enfermedad. ${ }^{3}$ La base multifactorial de la ECN incluye inmadurez intestinal, disbiosis microbiana, inestabilidad circulatoria del tubo intestinal e isquemia., ${ }^{2,3,15}$ El tamaño pequeño para la edad gestacional, el puntaje bajo de Apgar, la ventilación mecánica invasiva, los antibióticos empíricos, la nutrición con leche maternizada y el CAP son algunas de las manifestaciones clínicas asociadas con la progresión de la enfermedad. 3,6 En este estudio, se identificó que el CAP con repercusión hemodinámica y el puntaje bajo de Apgar fueron la causa principal de la aparición de ECN y de sus consecuencias graves (perforación intestinal). Ambos resultados confirman que la isquemia intestinal y la inestabilidad circulatoria son aspectos importantes de la ruta inflamatoria en la aparición de la ECN y las perforaciones intestinales.

También se demostró la función de la disbiosis microbiana en la lesión de las mucosas en la ECN. ${ }^{16,17}$ Para que el recién nacido prematuro logre un funcionamiento intestinal saludable, la maduración intestinal exige una relación comensal con la microbiota natural. Después del nacimiento, los microorganismos colonizan el tubo digestivo más rápidamente en el parto vaginal que en el parto por cesárea. Aunque no haya estudios que confirmen el papel de la cesárea en la aparición de la ECN, existen evidencias que sugieren que la

TABLA 1. Modelo de regresión multivariado para la aparición de ECN*

\begin{tabular}{lcccc}
\hline & & \multicolumn{2}{c}{ IC del 95 \% para Exp(B) } \\
& Exp (B) & Límite inferior & Límite superior & $p$ \\
\hline CAP & 4,715 & 2,717 & 8,183 & $<0,001$ \\
Primera alimentación enteral & $\mathbf{6 , 5 1 5}$ & $\mathbf{5 , 0 1 1}$ & $\mathbf{8 , 4 7 0}$ & $<\mathbf{0 , 0 0 1}$ \\
SDR & 3,538 & 2,238 & 5,594 & $<\mathbf{0 0 1}$ \\
Cesárea & 2,031 & 1,182 & 3,490 & $\mathbf{0 , 0 1 0}$ \\
Tipo de concepción: TRA & 1,434 & 0,791 & 2,600 & 0,235 \\
Puntaje de Apgar bajo al minuto 5 & 0,931 & 0,534 & 1,624 & 0,801 \\
Corticoesteroides posnatales & 0,900 & 0,454 & 1,782 & $0,762^{*}$ \\
\hline
\end{tabular}

La aparición de ECN hace referencia a la suma total de pacientes con sospecha de ECN y ECN avanzada.

El modelo no incluyó la edad gestacional ni el peso al nacer debido a una importante colinealidad.

$\mathrm{R}^{2}$ de Nagelkerke del modelo: 0,673; sensibilidad: $81,5 \%$; especificidad: 97,2\%.

Abreviaturas: CAP: conducto arterial persistente; SDR: síndrome de dificultad respiratoria; TRA: técnicas de reproducción asistida.

TABLA 2. Modelo de regresión logística multivariado para las perforaciones intestinales

\begin{tabular}{|c|c|c|c|c|}
\hline & \multirow[b]{2}{*}{$\operatorname{Exp}(B)$} & \multicolumn{2}{|c|}{ IC del $95 \%$ para $\operatorname{Exp}(B)$} & \multirow[b]{2}{*}{$p$} \\
\hline & & Límite inferior & Límite superior & \\
\hline Puntaje de Apgar bajo al minuto 5 & 2,748 & 1,100 & 6,866 & 0,031 \\
\hline CAP con repercusión hemodinámica & 0,471 & 0,173 & 1,285 & 0,142 \\
\hline Hemorragia intraventricular & 0,701 & 0,286 & 1,721 & 0,439 \\
\hline Ventilación mecánica invasiva & 1,799 & 0,464 & 6,969 & 0,395 \\
\hline Peso al nacer & 0,989 & 0,985 & 0,993 & $<0,001$ \\
\hline
\end{tabular}

R2 de Nagelkerke del modelo: 0,618; sensibilidad: 79,6\%; especificidad: 95,8\%. 
alteración de la flora intestinal podría participar en el mecanismo fisiopatológico. ${ }^{7,16-18}$ La repercusión de la cesárea en la aparición de la enfermedad fue evidente solo en los pacientes con ECN avanzada. Con este resultado, se ha verificado la necesidad de realizar investigaciones avanzadas de la microbiota para explorar la causalidad de la cesárea en la ECN. ${ }^{15}$ No obstante, para evitar esta enfermedad fatal, se debe concebir la idea de promover el parto vaginal en el nacimiento de prematuros, teniendo en cuenta los pros y los contras para el estado de salud de los recién nacidos. Esta hipótesis es especialmente importante para países como Turquía, México, Chile y Corea, ya que se clasificaron como los cuatro países con las tasas más altas de cesárea entre los países de la Organización para la Cooperación y el Desarrollo Económicos (OECD). ${ }^{19}$

La ECN es inversamente proporcional a la edad gestacional y al peso al nacer. ${ }^{6,7}$ La incidencia es del $12 \%$ para los pesos al nacer de 500 a 750 gramos, y hay una disminución del $3 \%$ por cada aumento de 250 gramos en el peso al nacer. ${ }^{20}$ Es importante mencionar el aumento global en la aparición de ECN en los recién nacidos de menos de 1000 gramos de este estudio. Suponemos que la discrepancia con los informes anteriores se debe a la mencionada ocupación de la unidad con traslados posnatales y al uso de los recursos de enfermería. La atención multidisciplinaria brindada por el centro del estudio la convierte en una de las UCIN que recibe más derivaciones, y a muchos recién nacidos prematuros de otros centros los trasladan a nuestra unidad cuando hay un deterioro clínico o se necesita una intervención quirúrgica. Por este motivo, consideramos que los números no reflejan la incidencia real de la ECN. Además, ser un centro receptor de tantas derivaciones tiene la desventaja de la sobreocupación de pacientes, donde se alcanza el $100 \%$ o más de la capacidad del lugar. Si bien la atención neonatal de los pacientes nuevos se brinda en camas diferentes, existe el riesgo de que los recursos de enfermería no sean suficientes, lo que es un reconocido factor predictivo de resultados adversos en los neonatos prematuros. ${ }^{21,22}$ La mejora de los servicios de enfermería ayudaría a aumentar la calidad de la atención perinatal. De esta forma, el pronóstico de los recién nacidos con bajo peso al nacer sería más promisorio en las UCIN de los países con ingresos medios y bajos.

La nutrición enteral temprana con leche materna es crucial para el crecimiento de los prematuros. ${ }^{1} \mathrm{Su}$ administración reduce la ECN, evita la atrofia vellosa y estimula el peristaltismo intestinal y la microbiota. ${ }^{2}$ La opinión mayoritaria internacional es que se debe iniciar la nutrición trófica de forma temprana y alcanzar la nutrición enteral total al cabo de una o dos semanas, según el peso al nacer. ${ }^{4} \mathrm{El}$ tiempo hasta alcanzar la alimentación enteral total se ha registrado entre 10 y 34 días en diferentes estudios, y se lo consideró un marcador indirecto de la tolerancia gastrointestinal. ${ }^{3,4} \mathrm{Sin}$ embargo, las prácticas de alimentación de los neonatos con muy bajo peso al nacer son considerablemente heterogéneas. ${ }^{23}$ Existen datos controvertidos sobre el aumento lento o rápido del volumen de ingesta diaria. Recientemente, en la base de datos Cochrane 2017, se ha registrado que el riesgo de ECN no se reduce con el aumento lento o rápido de dicho volumen. ${ }^{24} \mathrm{El}$ protocolo de nutrición de nuestra institución consiste en la nutrición trófica temprana con leche materna (si no la hubiera, con leche maternizada para prematuros, sin diluir), que se aumenta a diario en función de la tolerancia gastrointestinal. Lamentablemente, la ausencia de datos nutricionales de los traslados posnatales ha hecho difícil que se evalúe la influencia de la ingesta diaria (aumento de volumen lento o rápido) en la aparición de ECN. En su lugar, se analizó el momento de la primera alimentación enteral en relación con la aparición de ECN. Cada día de retraso en la administración de nutrición enteral ha aumentado 6,515 veces la probabilidad de contraer la enfermedad. Sin embargo, no fue evidente un resultado similar en el caso de las perforaciones intestinales. La información acerca de este tema es contradictoria: dos estudios recientes concluyeron que no hay relación entre el momento de la primera alimentación y la ECN (o la gravedad de la enfermedad), 3,6 mientras que Moss y cols. han demostrado la relación entre el retraso de la alimentación enteral y el daño intestinal y la aparición de ECN. ${ }^{25}$ Aparentemente, el retraso en la nutrición enteral causado por una dinámica cardiorrespiratoria inestable en los primeros días de vida anticipa futuros problemas intestinales del neonato, pero el proceso patológico de la perforación intestinal es más complejo de lo que se pensaba.

Las investigaciones han intentado crear paneles predictivos para identificar los casos de ECN avanzada y la necesidad de una intervención quirúrgica. ${ }^{26}$ En las perforaciones intestinales, se han hallado alteraciones como trombocitopenia, recuento anómalo de leucocitos y niveles elevados de PCR y glucemia. ${ }^{9}$ El análisis para predecir las perforaciones intestinales ha señalado la existencia de trombocitopenia, recuento elevado de leucocitos y acidosis metabólica de forma inicial en el proceso de deterioro, pero no quedó en claro si las alteraciones consiguientes en los análisis estuvieron acompañadas o no de infección concomitante (sepsis). Aunque las herramientas de diagnóstico no hayan podido predecir los casos de sospecha de ECN, parecen proporcionar los signos de advertencia iniciales para orientar a los profesionales de la salud en el cálculo de las 
consecuencias graves de la ECN.

La incidencia de la mortalidad fue coherente con las publicaciones anteriores, con una cifra del $10 \%$ al $40 \%$ (que llega hasta el $50 \%$ para la ECN con intervención quirúrgica). ${ }^{3,27}$ La probabilidad de mortalidad para la suma total de pacientes con ECN fue de 2,192 y aumentó en presencia de perforaciones intestinales.

Las limitaciones del estudio incluyen el diseño retrospectivo y unicéntrico. Otra de las limitaciones es la ausencia de datos sobre las ingestas diarias y los intervalos de alimentación. Los análisis diagnósticos se limitaron a las primeras etapas de los síntomas intestinales iniciales y no se obtuvieron las mediciones de laboratorio posteriores (el alcance de este artículo no incluía medir la eficacia predictiva de los resultados de laboratorio). A pesar de las limitaciones, puede destacarse como fortaleza de este estudio la naturaleza controlada y el amplio conjunto de datos, que incluye 1428 recién nacidos prematuros de una UCIN con gran cantidad de derivaciones. Además, en el diseño del estudio se incluyó cada etapa, desde los casos leves hasta la ECN avanzada y las perforaciones intestinales.

\section{CONCLUSIÓN}

En este estudio, la frecuencia de la ECN fue del 18,28\%. De los neonatos afectados, el $18 \%$ presentó perforación intestinal. El CAP con repercusión hemodinámica y el retraso en el inicio de la primera alimentación enteral aumentaron los riesgos de aparición de ECN, y el puntaje de Apgar bajo al minuto 5 siguió siendo el único factor predictivo independiente de la perforación intestinal.

\section{REFERENCIAS}

1. Neu j. Necrotizing Enterocolitis: The Future. Neonatology. 2020; 10:1-5.

2. Caplan MS. Necrotizing Enterocolitis in Preterm Infants is Related to Enteral Feeding, But the Mechanisms Remain Uncertain and Have Changed Over Time. Curr Pediatr Rep. 2014; 2(4):241-7.

3. Knell J, Han SM, Jaksic T, Modi BP. Current Status of Necrotizing Enterocolitis. Curr Probl Surg. 2019;56(1):11-38.

4. Binchy Á, MooreZ, Patton D. Feeding Intervals in Premature Infants $\leq 1750 \mathrm{~g}$ : An Integrative Review. Adv Neonatal Care. 2018; 18(3):168-78.

5. Stoll BJ, Hansen NI, Bell EF, Walsh MC y cols. Trends in Care Practices, Morbidity, and Mortality of Extremely Preterm Neonates, 1993-2012. JAMA. 2015;314(10):1039-51.

6. Adams M, Bassler D, Darlow BA, Lui K y cols. Preventive strategies and factors associated with surgically treated necrotising enterocolitis in extremely preterm infants: an international unit survey linked with retrospective cohort data analysis. BMJ Open. 2019; 9(10):e031086.

7. Denning NL, Prince JM. Neonatal intestinal dysbiosis in necrotizing enterocolitis. Mol Med. 2018; 24(1):4.

8. Overman RE, Criss CN, Gadepalli SK. Necrotizing enterocolitis in term neonates: A different disease process? J Pediatr Surg. 2019; 54(6):1143-6.

9. Yu L, Tian J, Zhao X, Cheng P y cols. Bowel Perforation in Premature Infants with Necrotizing Enterocolitis: Risk Factors and Outcomes. Gastroenterol Res Pract. 2016; 2016:6134187.

10. Hull MA, Fisher JG, Gutierrez IM, Jones BA y cols. Mortality and management of surgical necrotizing enterocolitis in very low birth weight neonates: a prospective cohort study. J Am Coll Surg. 2014; 218(6):1148-55.

11. Gordon P, Attridge J. Understanding Clinical Literature Relevant to Spontaneous Intestinal Perforations. Am J Perinatol. 2009; 26(04):309-16.

12. Vermont Oxford Network. Manual of Operations: Part 2 Data Definitions \& Infant Data Forms Vermont Oxford Network Release 23.2. 2019:101. [Fecha de acceso: 17 de enero de 2020]. Disponible en https: / / vtoxford.zendesk. com / hc/ en-us / articles / 360013115393-2019-Manual-ofOperations-Part-2-Release-23-2-PDF

13. Kültürsay N, Bilgen H, Türkyılmaz C. Turkish Neonatal Society guideline on enteral feeding of the preterm infant. Turk Pediatri Ars. 2018; 53(1):109-18.

14. Wang AY, Chughtai AA, LuiK, Sullivan EA. Morbidity and mortality among very preterm singletons following fertility treatment in Australia and New Zealand, a population cohort study. BMC Pregnancy Childbirth. 2017; 17(1):50.

15. Berkhout DJC, Klaassen P, Niemarkt HJ, de Boode WP y cols. Risk Factors for Necrotizing Enterocolitis: A Prospective Multicenter Case-Control Study. Neonatology 2018; 114(3):277-84.

16. Warner BB, Deych E, Zhou Y, Hall-Moore C y cols. Gut bacteria dysbiosis and necrotising enterocolitis in very low birthweight infants: a prospective case-control study. Lancet. 2016; 387(10031):1928-36.

17. Torrazza RM, Neu J. The Altered Gut Microbiome and Necrotizing Enterocolitis. Clin Perinatol. 2013;40(1):93-108.

18. Hällström M, Eerola E, Vuento R, Janas M, Tammela O. Effects of mode of delivery and necrotising enterocolitis on the intestinal microflora in preterm infants. Eur J Clin Microbiol Infect Dis. 2004; 23(6):463-70.

19. OECD. Health at a Glance 2019: OECD indicators. Paris: OECD Publishing, 2019. [Fecha de acceso: 17 de enero de 2020]. Disponibleen https: / / doi.org/10.1787/4dd50c09-en

20. Horbar JD, Edwards EM, Greenberg LT, Morrow KA y cols. Variation in Performance of Neonatal Intensive Care Units in the United States. JAMA Pediatr. 2017; 171(3):e164396.

21. Beltempo M, Lacroix G, Cabot M, Blais R, Piedboeuf B. Association of nursing overtime, nurse staffing and unit occupancy with medical incidents and outcomes of very preterm infants. J Perinatol. 2018; 38(2):175-80.

22. Watson SI, Arulampalam W, Petrou S, Marlow N y cols. The effects of a one-to-one nurse-to-patient ratio on the mortality rate in neonatal intensive care: a retrospective, longitudinal, population-based study. Arch Dis Child Fetal Neonatal Ed. 2016; 101(3):F195-200.

23. Bora R, Murthy NB. In resource limited areas complete enteral feed in stable very low birth weight infants (1000$1500 \mathrm{~g}$ ) started within $24 \mathrm{~h}$ of life can improve nutritional outcome. J Matern Neonatal Med. 2017; 30(21):2572-7.

24. Oddie SJ, Young L, Mcguire W. Slow advancement of enteral feed volumes to prevent necrotising enterocolitis in very low birth weight infants. Cochrane Database Syst Rev. 2017; 8(8):CD001241.

25. Moss RL, Kalish LA, Duggan C, Johnston P y cols. Clinical parameters do not adequately predict outcome in necrotizing enterocolitis: a multi-institutional study. $J$ Perinatol. 2008; 28(10):665-74.

26. Tepas JJ 3rd, Leaphart CL, Plumley D, Sharma R y cols. Trajectory of Metabolic Derangement in Infants with Necrotizing Enterocolitis Should Drive Timing and Technique of Surgical Intervention. J Am Coll Surg. 2010; 210(5):847-52.

27. Yee WH, Soraisham AS, Shah VS, Aziz K y cols. Incidence and Timing of Presentation of Necrotizing Enterocolitis in Preterm Infants. Pediatrics. 2012; 129(2):e298-e304. 


\section{ANEXO 1.}

Datos demográficos generales de la población

\begin{tabular}{|c|c|c|c|c|c|c|c|c|}
\hline & \multirow{2}{*}{$\begin{array}{c}\text { Total } \\
(\mathrm{n}=1428)\end{array}$} & \multirow{2}{*}{$\begin{array}{l}\text { Grupo de } \\
\text { referencia } \\
(n=1167)\end{array}$} & \multicolumn{2}{|c|}{ Aparición de ECN } & \multirow[b]{2}{*}{$p$} & \multirow[b]{2}{*}{$p 1^{\alpha}$} & \multirow[b]{2}{*}{$p 2^{\alpha}$} & \multirow[b]{2}{*}{$p 3^{\alpha}$} \\
\hline & & & $\begin{array}{l}\text { Sospecha } \\
\text { de ECN } \\
(n=140)\end{array}$ & $\begin{array}{c}\text { ECN } \\
\text { avanzada } \\
(n=121)\end{array}$ & & & & \\
\hline Edad materna (años) ${ }^{*}$ & $29(25-34)$ & $30(25-34)$ & $27(24-33,75)$ & $28(24-28)$ & 0,001 & 0,005 & 0,058 & 0,999 \\
\hline Patología materna & $360(25,2 \%)$ & $300(25,7 \%)$ & $29(20,7 \%)$ & $31(25,6 \%)$ & 0,435 & 0198 & 0,983 & 0,348 \\
\hline Madre con DG & $76(5,3 \%)$ & $65(5,6 \%)$ & $5(3,6 \%)$ & $5(5 \%)$ & 0,599 & 0,321 & 0,779 & 0,578 \\
\hline Afecciones de la placenta & a $18(1,3 \%)$ & $14(1,2 \%)$ & - & $4(3,3 \%)$ & 0,212 & - & 0,081 & - \\
\hline Preeclampsia & $219(15,3 \%)$ & $183(15,7 \%)$ & $20(14,3 \%)$ & $16(13,2 \%)$ & 0,725 & 0,667 & 0,476 & 0,804 \\
\hline RPM & $70(4,9 \%)$ & $57(4,9 \%)$ & $9(6,4 \%)$ & $4(3,3 \%)$ & 0,508 & 0,439 & 0,430 & 0,248 \\
\hline PEG & $39(2,7 \%)$ & $28(2,4 \%)$ & $8(5,7 \%)$ & $3(2,5 \%)$ & 0,076 & 0,033 & 0,573 & 0,195 \\
\hline \multicolumn{9}{|l|}{ Gestación múltiple } \\
\hline Mellizos & $194(13,6 \%)$ & $154(13,2 \%)$ & $25(17,9 \%)$ & $15(12,4 \%)$ & 0,291 & 0,130 & 0,804 & 0,222 \\
\hline Trillizos & $26(1,8 \%)$ & $15(1,3 \%)$ & $2(1,4 \%)$ & $9(7,4 \%)$ & 0,001 & 0,559 & $<0,001$ & 0,016 \\
\hline \multicolumn{9}{|l|}{ Tipo de parto } \\
\hline Cesárea & $1145(80,2 \%)$ & $916(78,5 \%)$ & $118(84,3 \%)$ & $111(91,7 \%)$ & 0,001 & 0,111 & 0,001 & 0,067 \\
\hline Parto vaginal & $283(19,8 \%)$ & $251(21,5)$ & $22(15,7 \%)$ & $10(19,8 \%)$ & & & & \\
\hline \multicolumn{9}{|l|}{ Tipo de concepción } \\
\hline TRA & $140(24,9 \%)$ & $80(6,9 \%)$ & $24(17,1 \%)$ & $36(29,8 \%)$ & $<0,001$ & $<0,001$ & $<0,001$ & 0,016 \\
\hline Recién nacido derivado & $514(36,0 \%)$ & $417(35,7 \%)$ & $45(32,1 \%)$ & $52(43 \%)$ & 0,174 & 0,401 & 0,115 & 0,071 \\
\hline Traslado prenatal & $117(8,2 \%)$ & $97(8,3 \%)$ & $6(4,3 \%)$ & $14(11,6 \%)$ & 0,095 & 0,094 & 0,226 & 0,027 \\
\hline \multicolumn{9}{|l|}{ Sexo } \\
\hline Femenino, $(\%)$ & $631(44,2 \%)$ & $512(43,9 \%)$ & $59(42,1 \%)$ & $60(49,6 \%)$ & 0,424 & 0,697 & 0,229 & 0,229 \\
\hline Masculino, $(\%)$ & $797(55,8 \%)$ & $655(56,1 \%)$ & $81(57,9 \%)$ & $61(50,4 \%)$ & & & & \\
\hline EG, semanas* & $32(30-34)$ & $32(32-34)$ & $29(27-31)$ & $26(25-27)$ & $<0,001$ & $<0,001$ & $<0,001$ & $<0,001$ \\
\hline$\leq 27$ semanas & $191(13,4 \%)$ & $59(5,1 \%)$ & $40(28,6 \%)$ & $92(76 \%)$ & & & & \\
\hline 28 - 30 semanas & $215(15,1 \%)$ & $134(11,5 \%)$ & $56(40 \%)$ & $25(20,7 \%)$ & & & & \\
\hline $31-33$ semanas & $508(35,6 \%)$ & $467(40 \%)$ & $37(26,4 \%)$ & $4(3,3 \%)$ & & & & \\
\hline $34-36$ semanas & $514(36 \%)$ & $507(43,4 \%)$ & $7(5 \%)$ & - & & & & \\
\hline Peso al nacer, gramos* & $\begin{array}{c}1760 \\
(1274-1909,2)\end{array}$ & $\begin{array}{c}1835 \\
(1620-1934)\end{array}$ & $\begin{array}{c}1120 \\
(975-1261)\end{array}$ & $\begin{array}{c}830 \\
(680-900)\end{array}$ & $<0,001$ & $<0,001$ & $<0,001$ & 0,003 \\
\hline$<750 \mathrm{~g}$ & $136(9,5 \%)$ & $76(6,5 \%)$ & $5(3,6 \%)$ & $55(45,4 \%)$ & & & & \\
\hline $751-1000 \mathrm{~g}$ & $147(10,3 \%)$ & $58(5 \%)$ & $40(28,6 \%)$ & $49(40,5 \%)$ & & & & \\
\hline $1001-1250 \mathrm{~g}$ & $132(9,2 \%)$ & $82(7 \%)$ & $42(30 \%)$ & $8(6,6 \%)$ & & & & \\
\hline $1251-1500 \mathrm{~g}$ & $147(10,3 \%)$ & $114(9,8 \%)$ & $28(20 \%)$ & $5(4,1 \%)$ & & & & \\
\hline $1501-2000 \mathrm{~g}$ & $665(46,6 \%)$ & $636(54,5 \%)$ & $25(17,9 \%)$ & $4(3,3 \%)$ & & & & \\
\hline$>2000 \mathrm{~g}$ & $201(14,1 \%)$ & $201(17,2 \%)$ & - & - & & & & \\
\hline
\end{tabular}

* Mediana, (intervalo intercuartílico); $\alpha$ : diferencia de las variables numéricas entre los tres grupos obtenida mediante el análisis de Kruskal-Wallis y comparaciones por pares mediante la prueba de Tamhane; $p 1$ : diferencia entre el grupo de referencia e intolerancia a la alimentación; $p 2$ : diferencia entre el grupo de referencia y el grupo de ECN; $p 3$ : diferencia entre el grupo de ECN e intolerancia a la alimentación.

Abreviaturas: ECN: enterocolitis necrosante; DG: diabetes gestacional; RPM: rotura prematura de membranas;

PEG: pequeño para la edad gestacional; TRA: técnicas de reproducción asistida; EG: edad gestacional;

Recién nacido derivado: nació en otro establecimiento y lo trasladaron al centro del estudio debido a problemas relacionados con la prematuridad. 


\section{ANEXO 2.}

\section{Desenlace clínico de la población del estudio}

\begin{tabular}{|c|c|c|c|c|c|c|c|c|}
\hline & \multirow{2}{*}{$\begin{array}{c}\text { Total } \\
(\mathrm{n}=1428)\end{array}$} & \multirow{2}{*}{$\begin{array}{l}\text { Grupo de } \\
\text { referencia } \\
(n=1167)\end{array}$} & \multicolumn{2}{|c|}{ Aparición de ECN } & \multirow[b]{2}{*}{$p$} & \multirow[b]{2}{*}{$p 1^{\alpha}$} & \multirow[b]{2}{*}{$p 2$} & \multirow[b]{2}{*}{$p 3$} \\
\hline & & & $\begin{array}{l}\text { Sospecha } \\
\text { de ECN } \\
(n=140)\end{array}$ & $\begin{array}{c}\mathrm{ECN} \\
\text { avanzada } \\
(\mathrm{n}=121)\end{array}$ & & & & \\
\hline Corticoesteroides prenatales & $847(59,3 \%)$ & $714(61,2 \%)$ & $77(55 \%)$ & $56(46,3 \%)$ & 0,004 & 0,157 & 0,001 & 0,160 \\
\hline Corticoesteroides posnatales & $116(8,1 \%)$ & $54(4,6 \%)$ & $19(13,6 \%)$ & $43(35,5 \%)$ & $<0,001$ & $<0,001$ & $<0,001$ & $<0,001$ \\
\hline Puntaje de Apgar al minuto 5 & $184(12,9 \%)$ & $129(11,1 \%)$ & $15(10,7 \%)$ & $40(33,1 \%)$ & $<0,001$ & 0,903 & $<0,001$ & $<0,001$ \\
\hline Reanimación en el nacimiento & $201(14,1 \%)$ & $158(13,5 \%)$ & $21(15 \%)$ & $22(18,2 \%)$ & 0,356 & 0,635 & 0,161 & 0,490 \\
\hline SDR & $233(16,3 \%)$ & $99(8,5 \%)$ & $49(35 \%)$ & $85(70,2 \%)$ & $<0,001$ & $<0,001$ & $<0,001$ & $<0,001$ \\
\hline VM invasiva & $403(28,2 \%)$ & $236(20,2 \%)$ & $64(45,7 \%)$ & $103(85,1 \%)$ & $<0,001$ & $<0,001$ & $<0,001$ & $<0,001$ \\
\hline Duración de la VM (días)* & $6(4-9)$ & $4,5(3-8)$ & $6(4-8)$ & $9(7-16)$ & $<0,001$ & 0,123 & $<0,001$ & $<0,001$ \\
\hline PPC & $680(47,6 \%)$ & $551(47,2 \%)$ & $57(40,7 \%)$ & $72(59,5 \%)$ & 0,008 & 0,145 & 0,010 & 0,002 \\
\hline Duración de la PPC (días)* & $6(3-11)$ & $4(2-8)$ & $9(5-12,75)$ & $12,5(8-18)$ & $<0,001$ & $<0,001$ & $<0,001$ & 0,999 \\
\hline Sepsis & $124(8,7 \%)$ & $62(5,3 \%)$ & $22(15,7 \%)$ & $40(33,1 \%)$ & $<0,001$ & $<0,001$ & $<0,001$ & 0,001 \\
\hline CAP $(\%)$ & $154(1$ & $52(4$, & $52(37,1 \%)$ & $50(41,3 \%)$ & $<0,001$ & $<0,001$ & $<0,001$ & 0,490 \\
\hline CC que no sea CAP & $74(5,2$ & $62(5,3$ & $3(2,1 \%)$ & $9(7,4 \%)$ & 0,140 & 0,103 & 0,330 & 0,059 \\
\hline HIV & $114(8,0 \%)$ & $35(3,0 \%)$ & $22(15,7 \%)$ & $57(47,1 \%)$ & $<0,001$ & $<0,001$ & $<0,001$ & $<0,001$ \\
\hline \multicolumn{9}{|c|}{ Resultado de la nutrición } \\
\hline Primera alimentación enteral (día) & )$^{*} 1(1-2)$ & $1(1-2)$ & $3(2-3)$ & $3(2-4)$ & $<0,001$ & $<0,001$ & $<0,001$ & 0,007 \\
\hline $\begin{array}{l}\text { Leche maternizada para } \\
\text { prematuros }\end{array}$ & $297(20,8 \%)$ & $234(20,1 \%)$ & $32(22,9 \%)$ & $31(25,6 \%)$ & 0,292 & 0,436 & 0,149 & 0,603 \\
\hline $\begin{array}{l}\text { Edad al momento de la aparición } \\
\text { de los síntomas (día) }\end{array}$ & $13(11-16)$ & 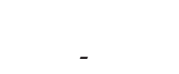 & $13(11-14)$ & $18(16-20)$ & $<0,001$ & - & - & $<0,001$ \\
\hline Duración del ayuno (días)* & $8(5-13)$ & - & $6(5-8)$ & $13(11-16)$ & $<0,001$ & - & - & $<0,001$ \\
\hline Nutrición parenteral (días) & $16(11-20)$ & $10(8-11)$ & $20(18-25)$ & $32(27-35)$ & $<0,001$ & $<0,001$ & $<0,001$ & $<0,001$ \\
\hline Estadía en la UCIN (días)* & $16(9-36)$ & $15(8-23)$ & $53(41-66,75)$ & $73(27-97)$ & $<0,001$ & $<0,001$ & $<0,001$ & 0,002 \\
\hline Mortalidad & $129(9,0 \%)$ & $89(7,6 \%)$ & $7(5,0 \%)$ & $33(27,3 \%)$ & $<0,001$ & 0,260 & $<0,001$ & $<0,001$ \\
\hline \multicolumn{9}{|c|}{ Análisis clínicos } \\
\hline Hematocrito (\%) & $\begin{array}{c}42,3 \\
(34.4-50.5)\end{array}$ & $\begin{array}{c}45,2 \\
(354-511)\end{array}$ & $\begin{array}{c}44,4 \\
(351-50.5)\end{array}$ & $\begin{array}{c}41,2 \\
(328-47,2)\end{array}$ & & & & \\
\hline Leucocitos (x 109/1) & $\begin{array}{c}15,2 \\
(12,6-18,3)\end{array}$ & $\begin{array}{c}14,8 \\
(12,4-18,1)\end{array}$ & $\begin{array}{c}15,8 \\
(13,3-18,4)\end{array}$ & $\begin{array}{c}18,38 \\
(15,1-23,3)\end{array}$ & $<0,001$ & 0,159 & $<0,001$ & $<0,001$ \\
\hline Leucocitosis & $211(14,8 \%)$ & $152(13 \%)$ & $13(9,3 \%)$ & $46(38 \%)$ & $<0,001$ & 0,208 & $<0,001$ & $<0,001$ \\
\hline Leucopenia & $36(2,5 \%)$ & $30(2,6 \%)$ & $2(1,4 \%)$ & $4(3,3 \%)$ & 0,608 & - & - & - \\
\hline Trombocitos $\left(\times 10^{9} / 1\right)$ & 218 & 228,5 & 218,5 & 198 & & & & \\
\hline & $(175-294,5)$ & $(171-338,5)$ & $(179-275)$ & $(151-290)$ & 0,115 & 0,673 & 0,160 & 0,531 \\
\hline Trombocitopenia & $37(2,6 \%)$ & $14(1,2 \%)$ & $4(2,9 \%)$ & $19(15,7 \%)$ & 0,001 & 0,484 & 0,003 & $<0,001$ \\
\hline PCR (mg/dl) & 1,1 & 0,96 & 1,19 & 1,02 & & & & \\
\hline & $(0,69-1,86)$ & $(0,69-1,62)$ & $(0,75-1,97)$ & $(1,00-1,99)$ & 0,310 & 0,147 & 0,133 & 0,980 \\
\hline Acidosis metabólica & $122(8,5 \%)$ & $3(0,3 \%)^{\mathrm{a}}$ & $31(22,1 \%)^{b}$ & $88(72,7 \%)^{c}$ & $<0,001$ & $<0,001$ & $<0,001$ & $<0,001$ \\
\hline
\end{tabular}

* Mediana, (intervalo intercuartílico); estadística de las variables numéricas obtenida mediante el análisis de Kruskal-Wallis y comparaciones por pares mediante la prueba de Tamhane; $p 1$ : grupo de referencia - grupo con sospecha de ECN; $p 2$ : grupo de referencia - grupo con ECN avanzada; 3 : grupo con sospecha de ECN - grupo con ECN avanzada. Abreviaturas: ECN: enterocolitis necrosante; SDR: síndrome de dificultad respiratoria; VM: ventilación mecánica; PPC: presión positiva continua; CAP: conducto arterial persistente; CC: cardiopatía congénita; HIV: hemorragia intraventricular; UCIN: unidad de cuidados intensivos neonatales; leucocitosis: leucocitos $>20 \times 10^{9} /$ l; leucopenia: leucocitos $<5 \times 10^{9} /$ l; trombocitopenia: trombocitos $<100 \times 10^{9} /$ l; PCR: proteína C-reactiva; acidosis metabólica ( $\mathrm{pH}<7,35$ y déficit de base $>-5$ ). 
ANEXO 3.

Características demográficas y desenlace clínico de las perforaciones GI

\begin{tabular}{|c|c|c|c|c|}
\hline & \multicolumn{2}{|c|}{ Aparición de ECN } & \multirow[b]{2}{*}{ OR (IC del $95 \%$ ) } & \multirow[b]{2}{*}{$p$} \\
\hline & $\begin{array}{c}\text { Sin perforaciones } \\
(\mathrm{n}=214)\end{array}$ & $\begin{array}{c}\text { Perforaciones } \\
(n=47)\end{array}$ & & \\
\hline Sexo masculino & $119(55,6 \%)$ & $23(48,9 \%)$ & $1,307(0,695-2,460)$ & 0,406 \\
\hline Edad materna, (años)* & $28(24-34)$ & $27(23-32)$ & $0,984(0,935-1,036)$ & 0,539 \\
\hline Patología materna & $52(24,3 \%)$ & $8(17 \%)$ & $0,639(0,281-1,454)$ & 0,283 \\
\hline Madre con DG & $11(5,1 \%)$ & - & - & NA \\
\hline Afección de la placenta & $4(1,9 \%)$ & - & - & NA \\
\hline Preeclampsia & $31(14,5 \%)$ & $5(10,6 \%)$ & $0,703(0,258-1,915)$ & 0,489 \\
\hline RPM & $9(4,2 \%)$ & $4(8,5 \%)$ & $2,119(0,629-7,197)$ & 0,189 \\
\hline PEG & $10(4,7 \%)$ & $1(2,1 \%)$ & $0,443(0,55-3,551)$ & 0,444 \\
\hline TRA & $49(22,9 \%)$ & $11(23,4 \%)$ & $1,029(0,488-2,171)$ & 0,379 \\
\hline Cesárea & $187(87,4 \%)$ & $42(89,4 \%)$ & $1,213(0,441-3,334)$ & 0,708 \\
\hline Traslado prenatal & $12(5,6 \%)$ & $8(17 \%)$ & $3,453(1,325-9,000)$ & 0,014 \\
\hline Recién nacido derivado & $73(34,1 \%)$ & $24(51,1 \%)$ & $2,015(1,065-3,814)$ & 0,029 \\
\hline \multicolumn{5}{|l|}{ Gestación múltiple } \\
\hline Mellizos & $33(15,4 \%)$ & $7(14,9 \%)$ & $0,960(0,396-2,325)$ & 0,928 \\
\hline Trillizos & $6(2,8 \%)$ & $5(1,6 \%)$ & - & NA \\
\hline Edad gestacional, (semanas) & $27,96 \pm 2,26$ & $25,87 \pm 1,99$ & & 0,001 \\
\hline$\leq 27$ semanas & $98(47,7 \%)$ & $34(72,3 \%)$ & $2,872(1,436-5,744)$ & 0,003 \\
\hline $28-30$ semanas & $68(31,7 \%)$ & $13(27,7 \%)$ & $0,770(0,383-1,550)$ & 0,463 \\
\hline $31-33$ semanas & $41(19,2 \%)$ & - & - & NA \\
\hline $34-36$ semanas & $7(3,3 \%)$ & - & - & NA \\
\hline Peso al nacer (gramos) & $1021,75 \pm 206,06$ & $722,02 \pm 139,88$ & & $<0,001$ \\
\hline$<750 \mathrm{~g}$ & $34(15,9 \%)$ & $26(55,3 \%)$ & $12,010(5,760-25,080)$ & $<0,001$ \\
\hline $751-1000 \mathrm{~g}$ & $75(35,1 \%)$ & $14(29,9 \%)$ & $1,031(0,546-1,946)$ & 0,425 \\
\hline $1001-1250 \mathrm{~g}$ & $45(21 \%)$ & $5(10,6 \%)$ & - & 0,646 \\
\hline $1251-1500 \mathrm{~g}$ & $31(14,5 \%)$ & $2(4,3 \%)$ & - & 0,547 \\
\hline $1500-2000 \mathrm{~g}$ & $29(13,6 \%)$ & - & - & NA \\
\hline \multicolumn{5}{|l|}{ Desenlace clínico } \\
\hline Corticoesteroides prenatales & $113(52,8 \%)$ & $20(42,6 \%)$ & $0,662(0,350-1,252)$ & 0,203 \\
\hline Corticoesteroides posnatales & $43(20,1 \%)$ & $19(40,4 \%)$ & $2,699(1,379-5,282)$ & 0,003 \\
\hline Reanimación & $37(17,3 \%)$ & $6(12,8 \%)$ & $0,700(0,277-1,769)$ & 0,449 \\
\hline Puntaje de Apgar bajo al minuto 5 & $33(15,4 \%)$ & $22(46,8 \%)$ & $4,827(2,439-9,552)$ & $<0,001$ \\
\hline SDR & $97(45,3 \%)$ & $37(78,7 \%)$ & $4,463(2,111-9,435)$ & 0,001 \\
\hline VM invasiva & $123(57,5 \%)$ & $44(93,6 \%)$ & $10,851(3,267-36,045)$ & $<0,001$ \\
\hline Duración de la VM (días)* & $7(5-10)$ & $11(7,25-22,5)$ & $1,153(1,086-1,224)$ & $<0,001$ \\
\hline PPC & $115(53,7 \%)$ & $14(29,8 \%)$ & $0,365(0,185-0,721)$ & 0,003 \\
\hline Duración de la PPC (días)* & $13(9-18)$ & $21,5(15,5-26,75)$ & $1,114(1,048-1,184)$ & 0,001 \\
\hline Sepsis & $43(20,1 \%)$ & $19(40,4 \%)$ & $2,699(1,379-5,282)$ & 0,003 \\
\hline CÁP & $77(36 \%)$ & $25(53,2 \%)$ & $2,022(1,069-3,824)$ & 0,029 \\
\hline CC que no sea CAP $(\%)$ & $5(2,3 \%)$ & $7(14,9 \%)$ & - & \\
\hline NA & & & & \\
\hline HIV & $56(26,2 \%)$ & $23(48,9 \%)$ & $2,704(1,414-5,169)$ & 0,002 \\
\hline \multicolumn{5}{|l|}{ Nutrición } \\
\hline $\begin{array}{l}\text { Momento de la primera } \\
\text { alimentación enteral (día)* }\end{array}$ & & & & \\
\hline $\begin{array}{l}\text { alimentación enteral, (día)* } \\
\text { Leche maternizada sin diluir }\end{array}$ & $3(2-4)$ & $3(2-4)$ & $1,092(0,784-1,520)$ & 0,604 \\
\hline $\begin{array}{l}\text { Leche maternizada sin diluir } \\
\text { para prematuros }\end{array}$ & $48(22,4 \%)$ & $15(31,9 \%)$ & $1,621(0,811-3,240)$ & 0,169 \\
\hline Edad al momento de la aparición & & & & \\
\hline de los síntomas GI, (día) $)^{\frac{1}{x}}$ & $14(11-16)$ & $19(17-21)$ & $1,479(1,305-1,675)$ & $<0,001$ \\
\hline Duración del ayuno, (días)* & $10(7-12)$ & $16(13-18)$ & $1,679(1,439-1,958)$ & $<0,001$ \\
\hline Nutrición parenteral, (días)* & $16(14-17)$ & $34(32,5-35)$ & $1,230(1,121-1,350)$ & $<0,001$ \\
\hline \multicolumn{5}{|l|}{ Pruebas de diagnóstico } \\
\hline Hematocrito $(\%)^{\star}$ & $44,9(35,4-50,72)$ & $43,9(35,1-50,7)$ & $1,004(0,971-1,038)$ & 0,810 \\
\hline Leucocitosis & $34(15,9 \%)$ & $25(53,2 \%)$ & $6,016(3,047-11,877)$ & $<0,001$ \\
\hline Leucopenia & $4(1,9 \%)$ & $2(4,3 \%)$ & $2,33(0,415-13,131)$ & 0,295 \\
\hline Recuento de trombocitos $\left(\times 10^{9} / 1\right)$ & $211(178-286)$ & $194(92-290)$ & $0,997(0,994-1,001)$ & 0,086 \\
\hline Trombocitopenia & $10(4,7 \%)$ & $13(27,7 \%)$ & $7,800(3,169-19,200)$ & $<0,001$ \\
\hline $\operatorname{PCR}(\mathrm{mg} / \mathrm{dl})^{*}$ & $0,80(0,60-1,72)$ & $1,19(0,70-2,03)$ & $0,859(0,671-1,099)$ & 0,049 \\
\hline Acidosis metabólica & $72(33,6 \%)$ & $47(100 \%)$ & $1,653(1,429-1,911)$ & $<0,001$ \\
\hline
\end{tabular}

-Razón de posibilidades obtenida mediante regresión binaria si $p<0,05$; * mediana (intervalo intercuartílico);

NA: no aplicable debido a la poca cantidad de pacientes en el grupo.

-Leucopenia: leucocitos < $5 \times 10^{9} / 1$; leucocitosis: leucocitos $>20 \times 10^{9} / 1$; trombocitopenia: trombocitos < $100 \times 10^{9} / 1$.

Abreviaturas: DG: diabetes gestacional; RPM: rotura prematura de membranas; PEG: pequeño para la edad gestacional;

TRA: técnicas de reproducción asistida; EG: edad gestacional; SDR: síndrome de dificultad respiratoria; VM: ventilación mecánica;

PPC: presión positiva continua; CAP: conducto arterial persistente; CC: cardiopatía congénita; HIV: hemorragia intraventricular; leucocitosis: leucocitos $>20 \times 10^{9} / 1$; leucopenia: leucocitos $<5 \times 10^{9} / 1$; trombocitopenia: trombocitos $<100 \times 10^{9} / 1$;

PCR: proteína C-reactiva; acidosis metabólica ( $\mathrm{pH}<7,35$ y déficit de base $>-5$ ). 